\title{
The warped product of holomorphic Lie algebroids
}

\author{
Alexandru Ionescu and Gheorghe Munteanu
}

\begin{abstract}
We introduce the warped product of two holomorphic Finsler algebroids and we define a complex Finsler function on it. We study the Chern-Finsler connections of the bundles and of their product and we investigate their curvatures. We use the geometrical setting of the prolongations of the two bundles to obtain some similar and some different properties from the ones of the warped product of Finsler manifolds.
\end{abstract}

\section{Introduction}

Lie algebroids have been intensely studied in the past decades as a theory with special applications in mechanics, due to their property of unifying tangent bundles and Lie algebras. The study was started by Weinstein [34], who developed a generalized theory of Lagrangians on Lie algebroids and obtained the Euler-Lagrange equations using the structure of the dual of a Lie algebroid and Legendre transformations associated with a regular Lagrangian. He also raised a question on developing a similar formalism to Klein's in ordinary Lagrangian mechanics, without reference to the dual. The first answer was due to Martinez [25, 26], who developed earlier ideas of Klein [21] using prolongations of Lie algebroids, a setting introduced under another name by Higgins and Mackenzie in [12]. This approach was used to study almost

Key Words: Lie algebroid, warped product, prolongation bundle, nonlinear connection, Finsler bundle.

2010 Mathematics Subject Classification: Primary 17B66, 53B40, 53C60; Secondary 53D17.

Received: 08.05.2019.

Accepted: 30.06.2019. 
every aspect of the classical theory (vakonomic and nonholonomic constraints, Hamilton-Jacobi theory, controls, higher order systems), see also [27, 24, 1].

Another approach on Weinstein's problem uses the Tulczyjew triple structure $[11,10]$. The connection between the two geometrical settings was analyzed in [20]. Recent applications for Lie algebroids were given in optimal control, interpolation problems, trajectory planning any many more, see for instance $[24,9]$ and the numerous references therein.

The warped products in Finsler geometry are a new field of research, initiated in [22], motivated by relativistic models described by Asanov [4]. In Riemannian geometry, warped products were studied, for instance, in $[3,7,33]$. Some applications of warped products in cosmology were given in $[13,14]$.

Following the line of the study of holomorphic Lie algebroids and their prolongations, initiated in $[16,17,18,19]$, in the present paper we investigate the notion of warped product $E_{1} \times_{f} E_{2}$ of two algebroids $E_{1}$ and $E_{2}$.

The Finsler metric that we define on the product bundle $E_{1} \times_{f} E_{2}$ is not the only or the most general metric which can be considered. In fact, it is known from [8] that there is no canonical way of defining Finsler metrics on products of Finsler manifolds. We can extend this remark to products of Finsler bundles. There are warped product bundles, as the one considered here, named after warped manifolds, studied for instance in [22]. Then, there are doubly warped products, as the ones studied also for products of manifolds in $[30,23]$, where two warping functions are involved. This is in its turn an interesting case for a future study. Also, if the function $f$ depends also on the vector variables, then the product is called twisted. Such product of manifolds were studied, for instance, in [31]. A more general class of Finsler metrics on a product of two Finsler manifolds was studied in [35]. We consider that the (simply) warped product setting that we investigate in the paper can have applications in theoretical physics. We also found an example for applying the present study.

In the first section, we recall the necessary notions about holomorphic Lie algebroids with Finsler structures and their prolongations. Also, we give some properties of the Chern-Finsler connection of a Finsler algebroid, such as its torsions and curvatures, as well as the metric property.

In a separate subsection, we give the definitions of two important notions needed in the paper, namely, the gradient and the Hessian of a function on an algebroid. We also obtain some different properties of the Hessian in comparison to the one defined on Finsler bundles.

The second section introduces the main notion of the paper, the warped product of two holomorphic Finsler algebroids, which, as we prove, is in its turn a Finsler bundle.

In the third section, we study the relation between the Chern-Finsler con- 
nections of the algebroids and that of their product. We restrict our considerations on the vertical subbundles. Also, we investigate the vertical curvature on the warped product, to find that it has some similar properties to that obtained for horizontal fields on a Finsler manifold [22].

In the last section, we give an example of a warped product of algebroids with applications in gravitation and electromagnetism theories, using the standard algebroid $T^{\prime} M$.

\section{Complex Finsler structures on the prolongation alge- broid}

We recall from [17] the definition of a Finsler Lie algebroid $\pi: E \rightarrow M$, with the anchor map $\rho_{E}: E \rightarrow T^{\prime} M$. First, denote by $\widetilde{E}$ the open submanifold of $E$ consisting in the nonzero sections. In a local chart in $z \in M$, we have local coordinates $\left\{z^{k}\right\}_{k=\overline{1, n}}$ and a section on $E$ is $u=u^{\alpha} e_{\alpha}$, where $\left\{e_{\alpha}\right\}_{\alpha=\overline{1, m}}$ is a basis of local sections on $E$.

Definition 1. A complex Finsler structure $F$ on $E$ is a real-valued function $F: E \rightarrow \mathbb{R}$ satisfying the following conditions:

1) $F$ is $C^{\infty}$-class on $\widetilde{E}$;

2) $F(z, u) \geq 0$ and $F(z, u)=0$ iff $u=0$;

3) $F(z, \lambda u)=|\lambda|^{2} F(z, u)$ for all $\lambda \in \mathbb{C}$.

As in the case of complex vector bundles [2], we say that a Finsler structure $F$ is convex if the Hermitian matrix defined in our case by

$$
h_{\alpha \bar{\beta}}=\frac{\partial^{2} F}{\partial u^{\alpha} \partial \bar{u}^{\beta}}
$$

is positive-definite. In the following, we assume the convexity of $F$ and call the pair $(E, F)$ a complex Finsler Lie algebroid.

The most well-known linear connection in Finsler geometry is the ChernFinsler connection. Here, we briefly recall from [17] the Chern-Finsler connection of the Lie algebroid $E$.

On a complex vector bundle, the notion of "normal" complex linear connection does not make sense, due to the fact that the rules of change of the coefficients of a distinguished linear connection do not coincide in pairs, such as in the case of the holomorphic tangent bundle $T^{\prime} M$. This is well-known from [28]. Therefore, we have induced a Chern-Finsler linear connection on the prolongation $\mathcal{T} E$ starting from a vertical connection on $E$. 
Let us first recall the construction of the holomorphic prolongation $\mathcal{T}^{\prime} E$. For more details, see $[16,17,18]$. Let $E$ be a holomorphic Lie algebroid over a complex manifold $M$. Its holomorphic prolongation is defined using the tangent mapping $\pi_{*}^{\prime}: T^{\prime} E \rightarrow T^{\prime} M$ between the holomorphic tangent bundles of $E$ and $M$, respectively, and the holomorphic anchor map $\rho_{E}: E \rightarrow T^{\prime} M$. Define the subset $\mathcal{T}^{\prime} E$ of $E \times T^{\prime} E$ by $\mathcal{T}^{\prime} E=\left\{(e, v) \in E \times T^{\prime} E \mid \rho_{E}(e)=\right.$ $\left.\pi_{*}^{\prime}(v)\right\}$ and the mapping $\pi_{\mathcal{T}}^{\prime}: \mathcal{T}^{\prime} E \rightarrow E$, given by $\pi_{\mathcal{T}}^{\prime}(e, v)=\pi_{E}(v)$, where $\pi_{E}^{\prime}: T^{\prime} E \rightarrow E$ is the tangent projection. Then, $\left(\mathcal{T}^{\prime} E, \pi_{\mathcal{T}}^{\prime}, E\right)$ is a holomorphic vector bundle over $E$, of rank $2 m$.

The holomorphic Lie algebroid $E$ has a structure of holomorphic vector bundle. Let $E_{\mathbb{C}}$ be the complexified bundle of $E$ and $T_{\mathbb{C}} E=T^{\prime} E \oplus T^{\prime \prime} E$, its complexified tangent bundle. A similar idea to that of Martinez [25, 26] leads to the definition of the complexified prolongation $\mathcal{T}_{\mathbb{C}} E$ of $E$ as follows. We extend $\mathbb{C}$-linearly the tangent mapping $\pi_{*}^{\prime}: T^{\prime} E \rightarrow T^{\prime} M$ and the anchor $\rho_{E}: E \rightarrow T^{\prime} M$ to obtain $\pi_{*, \mathbb{C}}: T_{\mathbb{C}} E \rightarrow T_{\mathbb{C}} M$ and $\rho_{E, \mathbb{C}}: E_{\mathbb{C}} \rightarrow T_{\mathbb{C}} M$, respectively. If $\pi_{E, \mathbb{C}}: T_{\mathbb{C}} E \rightarrow E_{\mathbb{C}}$ is the tangent projection extended to the complexified spaces, then we can define the subset $\mathcal{T}_{\mathbb{C}} E$ of $E_{\mathbb{C}} \times T_{\mathbb{C}} E$ by

$$
\mathcal{T}_{\mathbb{C}} E=\left\{(e, v) \in E_{\mathbb{C}} \times T_{\mathbb{C}} E \mid \rho_{E, \mathbb{C}}(e)=\pi_{*, \mathbb{C}}(v)\right\}
$$

and the mapping $\pi_{\mathcal{T}, \mathbb{C}}: \mathcal{T}_{\mathbb{C}} E \rightarrow E_{\mathbb{C}}$ by $\pi_{\mathcal{T}, \mathbb{C}}(e, v)=\pi_{E, \mathbb{C}}(v)$. Thus, we obtain a complex vector bundle $\left(\mathcal{T}_{\mathbb{C}} E, \pi_{\mathcal{T}, \mathbb{C}}, E_{\mathbb{C}}\right)$ over $E_{\mathbb{C}}$. Also, the projection onto the second factor,

$$
\rho_{\mathcal{T}, \mathbb{C}}: \mathcal{T}_{\mathbb{C}} E \rightarrow T_{\mathbb{C}} E, \quad \rho_{\mathcal{T}, \mathbb{C}}(e, v)=v,
$$

is the anchor of a complex Lie algebroid over $E_{\mathbb{C}}$, called the complexified prolongation of $E$. Indeed, $\mathcal{T}^{\prime} E=E^{\prime} \times T^{\prime} E$ is a holomorphic product bundle and, since $\rho_{E, \mathbb{C}}=\rho^{\prime}+\rho^{\prime \prime}$ and $\pi_{*, \mathbb{C}}=\pi_{*}^{\prime}+\pi_{*}^{\prime \prime}$ are holomorphic mappings with $\rho_{E, \mathbb{C}}(e)=\pi_{*, \mathbb{C}}(v)$, then $\rho^{\prime}(\bar{e})=\pi_{*}^{\prime}(\bar{v})=0$. Hence, the complexified prolongation coincides with the complexification of the prolongation $\mathcal{T}^{\prime} E$ (as a complex manifold), that is $\mathcal{T}_{\mathbb{C}} E=\mathcal{T}^{\prime} E \oplus \mathcal{T}^{\prime \prime} E$, where $\mathcal{T}^{\prime \prime} E=\overline{\mathcal{T}^{\prime} E}=E^{\prime \prime} \times T^{\prime \prime} E$, with the required restrictions $\rho_{E}^{\prime}(e)=\pi_{*}^{\prime}(v)$ and its conjugate. We will further drop the index $\mathbb{C}$ and simply denote the prolongation bundle by $\mathcal{T} E$.

In [17], following the ideas from [2], we have introduced the Chern-Finsler nonlinear connection of the prolongation $\mathcal{T} E$. We use the abbreviations $\partial_{k}=$ $\frac{\partial}{\partial z^{k}}, \dot{\partial}_{\alpha}=\frac{\partial}{\partial u^{\alpha}}$ and their conjugates, and we denote by $\rho_{\alpha}^{k}=\rho_{\alpha}^{k}(z)$, the local holomorphic function coefficients of the anchor map $\rho_{E}$. If $F: E \rightarrow \mathbb{R}_{+}$is a Finsler function on $E$, namely it is homogeneous, and the complex Finsler metric tensor $h_{\alpha \bar{\beta}}=\dot{\partial}_{\alpha} \dot{\partial}_{\bar{\beta}} F$ is strictly pseudoconvex, then we consider the Chern-Finsler nonlinear connection on $E$

$$
N_{k}^{\beta}=h^{\bar{\sigma} \beta} \partial_{k} \dot{\partial}_{\bar{\sigma}} F
$$


and we obtain that

$$
N_{\alpha}^{\beta}=\rho_{\alpha}^{k} N_{k}^{\beta}
$$

are the coefficients of the Chern-Finsler nonlinear connection of the prolongation $\mathcal{T}^{\prime} E$.

Also in [17], we have considered the local basis of holomorphic sections in $\Gamma\left(\mathcal{T}^{\prime} E\right)$, denoted by $\left\{\mathcal{Z}_{\alpha}:=\left(e_{\alpha}, \rho_{\alpha}^{k} \partial_{k}\right), \mathcal{V}_{\alpha}:=\left(0, \dot{\partial}_{\alpha}\right)\right\}$ and their conjugates, $\left\{Z_{\bar{\alpha}}, \mathcal{V}_{\bar{\alpha}}\right\}$ in $\Gamma\left(\mathcal{T}^{\prime \prime} E\right)$. The dual basis will be denoted by $\left\{d \mathcal{Z}^{\alpha}, d \mathcal{V}^{\alpha}, d Z^{\bar{\alpha}}, d \mathcal{V}^{\bar{\alpha}}\right\}$.

With respect to the nonlinear connection (2), consider the adapted frames on the holomorphic prolongation $\mathcal{T}^{\prime} E$

$$
x_{\alpha}=z_{\alpha}-N_{\alpha}^{\beta} \nu_{\alpha}
$$

and its conjugate. The dual adapted frames of $\left\{x_{\alpha}, \mathcal{V}_{\alpha}\right\}$ are $\left\{d z^{\alpha}, \delta \mathcal{V}^{\alpha}:=\right.$ $\left.d \mathcal{V}^{\alpha}+N_{\beta}^{\alpha} d Z^{\beta}\right\}$.

In [19], we have proved that the anchor map of the prolongation algebroid sends the adapted frame $X_{\alpha}$ in an adapted frame on the complex algebroid $E$, that is, $\rho_{\mathcal{T}}\left(X_{\alpha}\right)=\delta_{\alpha}=: \rho_{\alpha}^{k} \delta_{k}$, where $\delta_{k}=\partial_{k}-N_{k}^{\alpha} \dot{\partial}_{\alpha}$. With respect to the nonlinear connection (2), the holomorphic prolongation $\mathcal{T}^{\prime} E$ decomposes into vertical and horizontal bundles, $\mathcal{T}^{\prime} E=H \mathcal{T}^{\prime} E \oplus V \mathcal{T}^{\prime} E$.

Also, a Chern-Finsler linear connection of type $(1,0)$ on $\mathcal{T}^{\prime} E$, [17], is given by the coefficients:

$$
L_{\alpha \beta}^{\gamma}=h^{\bar{\sigma} \gamma} \delta_{\beta}\left(h_{\alpha \bar{\sigma}}\right), \quad C_{\alpha \beta}^{\gamma}=h^{\bar{\sigma} \gamma} \dot{\partial}_{\beta}\left(h_{\alpha \bar{\sigma}}\right) .
$$

Its connection form is

$$
\omega_{\alpha}^{\gamma}=L_{\alpha \beta}^{\gamma} z^{\beta}+C_{\alpha \beta}^{\gamma} \delta \nu^{\beta}
$$

We note that the vertical connection coefficients satisfy $C_{\alpha \beta}^{\gamma}=C_{\beta \alpha}^{\gamma}$ and the horizontal ones, $L_{\alpha \beta}^{\gamma}=\dot{\partial}_{\alpha} N_{\beta}^{\gamma}$. Using these identities, we obtain the components of the torsion of the Chern-Finsler connection on $\mathcal{T} E$ :

$$
\begin{aligned}
& \mathcal{T}\left(x_{\alpha}, x_{\beta}\right)=\left(L_{\beta \alpha}^{\gamma}-L_{\alpha \beta}^{\gamma}-\mathcal{C}_{\alpha \beta}^{\gamma}\right) x_{\gamma}-\mathcal{R}_{\alpha \beta}^{\gamma} \mathcal{V}_{\gamma}, \\
& \mathcal{T}\left(x_{\alpha}, x_{\bar{\beta}}\right)=-\left(\delta_{\bar{\beta}} N_{\alpha}^{\gamma}\right) \mathcal{V}_{\gamma}+\left(\delta_{\alpha} N_{\bar{\beta}}^{\bar{\gamma}}\right) \mathcal{V}_{\bar{\gamma}}, \\
& \mathcal{T}\left(x_{\alpha}, \mathcal{V}_{\beta}\right)=-C_{\alpha \beta}^{\gamma} x_{\gamma}, \\
& \mathcal{T}\left(x_{\alpha}, \mathcal{V}_{\bar{\beta}}\right)=-\left(\dot{\partial}_{\bar{\beta}} N_{\alpha}^{\gamma}\right) \mathcal{V}_{\gamma}, \\
& \mathcal{T}\left(\mathcal{V}_{\alpha}, \mathcal{V}_{\beta}\right)=0, \\
& \mathcal{T}\left(\mathcal{V}_{\alpha}, \mathcal{V}_{\bar{\beta}}\right)=0 .
\end{aligned}
$$

On the prolongation $\mathcal{T} E$, the Hermitian metric structure is defined by

$$
\mathcal{G}=h_{\alpha \bar{\beta}} d z^{\alpha} \otimes d \bar{z}^{\beta}+h_{\alpha \bar{\beta}} \delta \mathcal{V}^{\alpha} \otimes \delta \overline{\mathcal{V}}^{\beta} .
$$


The Chern-Finsler connection (4) is metric with respect to the hermitian structure $\mathcal{G}$, i.e., it satisfies the identity:

$$
U \mathcal{G}(V, W)=\mathcal{G}\left(\mathcal{D}_{U} V, W\right)+\mathcal{G}\left(V, \mathcal{D}_{U} W\right)
$$

(see [17]). Also, as we will prove in the following, the Chern-Finsler connection verifies the Koszul formula on the vertical subbundle of the prolongation.

Lemma 1. Let $(E, F)$ be a holomorphic Finsler Lie algebroid with the ChernFinsler connection $\mathcal{D}$. For $U, V, W \in V \mathcal{T} E$, the following identity holds:

$$
\begin{aligned}
2 \mathcal{G}\left(\mathcal{D}_{U} V, W\right)=U \mathcal{G}(V, W) & +V \mathcal{G}(W, U)-W \mathcal{G}(U, V) \\
& -\mathcal{G}(U,[V, W])+\mathcal{G}(V,[W, U])+\mathcal{G}(W,[U, V]) .
\end{aligned}
$$

Proof. It consists of a simple computation, using the fact that the torsion of two vertical fields vanishes.

\subsection{The gradient and the Hessian of a function}

In [18], we have introduced the gradient of a function on the holomorphic prolongation $\mathcal{T}^{\prime} E$. Now, we are interested in the gradient defined on the bundle $\mathcal{T} E$, i.e., the operator $\nabla$ given by

$$
\mathcal{G}(Z, \nabla f)=Z f, \forall Z \in \mathcal{T} E .
$$

In coordinates, this reads

$$
\nabla f=h^{\bar{\beta} \alpha}\left(\delta_{\bar{\beta}} f\right) X_{\alpha}+h^{\bar{\beta} \alpha}\left(\delta_{\alpha} f\right) X_{\bar{\beta}}+h^{\bar{\beta} \alpha}\left(\dot{\partial}_{\bar{\beta}} f\right) \mathcal{V}_{\alpha}+h^{\bar{\beta} \alpha}\left(\dot{\partial}_{\alpha} f\right) \mathcal{V}_{\bar{\beta}} .
$$

In the following, we will consider the vertical part of the gradient, that is,

$$
\nabla^{v \bar{v}} f=h^{\bar{\beta} \alpha}\left(\dot{\partial}_{\bar{\beta}} f\right) \mathcal{V}_{\alpha}+h^{\bar{\beta} \alpha}\left(\dot{\partial}_{\alpha} f\right) \mathcal{V}_{\bar{\beta}}
$$

We now define the Hessian of a function in a classical manner and obtain some identities which differ slightly from the properties of the Hessian defined on a Finsler manifold.

Definition 2. The Hessian of a function $f$ with respect to the Chern-Finsler connection $\mathcal{D}$ on $\mathcal{T} E$ is the second covariant differential $\mathcal{H} f=\mathcal{D}(\mathcal{D} f)$.

Proposition 1. The Hessian $\mathcal{H} f$ satisfies the identities:

$$
\begin{aligned}
\mathcal{H} f(V, W) & =V W f-\left(\mathcal{D}_{V} W\right) f+\left(\mathcal{D}_{V^{v}} W^{v}+\mathcal{D}_{V^{\bar{v}}} W^{\bar{v}}\right) f \\
& =\mathcal{G}\left(\mathcal{D}_{V}\left(\nabla^{v \bar{v}} f\right), W\right)+\left(\mathcal{D}_{V^{v}} W^{v}+\mathcal{D}_{V^{\bar{v}}} W^{\bar{v}}\right) f,
\end{aligned}
$$

where $V=V^{\alpha} \mathcal{V}_{\alpha}+V^{\bar{\alpha}} \mathcal{V}_{\bar{\alpha}}, W=W^{\beta} \mathcal{V}_{\beta}+W^{\bar{\beta}} \mathcal{V}_{\bar{\beta}},\left(\mathcal{D}_{V^{v}} W^{v}\right) f=\left(\mathcal{D}_{V^{\alpha} \mathcal{V}_{\alpha}} W^{\beta} \mathcal{V}_{\beta}\right) f$ $=V^{\alpha}\left(\dot{\partial}_{\alpha} W^{\beta}\right)\left(\dot{\partial}_{\beta} f\right)$ and $\left(\mathcal{D}_{V^{\bar{v}}} W^{\bar{v}}\right) f$ is its conjugate. 
Proof. First,

$$
\begin{aligned}
\mathcal{H} f(V, W)=\mathcal{D}_{V} \mathcal{D}_{W} f & =V^{\alpha}\left(\dot{\partial}_{\alpha} W^{\beta}\right)\left(\dot{\partial}_{\beta} f\right)+V^{\alpha} W^{\beta}\left(\dot{\partial}_{\alpha} \dot{\partial}_{\beta} f\right)+V^{\alpha} W^{\bar{\beta}}\left(\dot{\partial}_{\alpha} \dot{\partial}_{\bar{\beta}} f\right) \\
& +V^{\bar{\alpha}} W^{\beta}\left(\dot{\partial}_{\bar{\alpha}} \dot{\partial}_{\beta} f\right)+V^{\bar{\alpha}}\left(\dot{\partial}_{\bar{\alpha}} W^{\bar{\beta}}\right)\left(\dot{\partial}_{\bar{\beta}} f\right)+V^{\bar{\alpha}} W^{\bar{\beta}}\left(\dot{\partial}_{\bar{\alpha}} \dot{\partial}_{\bar{\beta}} f\right) .
\end{aligned}
$$

Next,

$$
\begin{aligned}
V W f & =V^{\alpha}\left(\dot{\partial}_{\alpha} W^{\beta}\right)\left(\dot{\partial}_{\beta} f\right)+V^{\alpha} W^{\beta}\left(\dot{\partial}_{\alpha} \dot{\partial}_{\beta} f\right)+V^{\alpha}\left(\dot{\partial}_{\alpha} W^{\bar{\beta}}\right)\left(\dot{\partial}_{\bar{\beta}} f\right) \\
& +V^{\alpha} W^{\bar{\beta}}\left(\dot{\partial}_{\alpha} \dot{\partial}_{\bar{\beta}} f\right)+V^{\bar{\alpha}}\left(\dot{\partial}_{\bar{\alpha}} W^{\beta}\right)\left(\dot{\partial}_{\beta} f\right)+V^{\bar{\alpha}} W^{\beta}\left(\dot{\partial}_{\bar{\alpha}} \dot{\partial}_{\beta} f\right) \\
& +V^{\bar{\alpha}}\left(\dot{\partial}_{\bar{\alpha}} W^{\bar{\beta}}\right)\left(\dot{\partial}_{\bar{\beta}} f\right)+V^{\bar{\alpha}} W^{\bar{\beta}}\left(\dot{\partial}_{\bar{\alpha}} \dot{\partial}_{\bar{\beta}} f\right)
\end{aligned}
$$

and

$$
\begin{aligned}
\left(\mathcal{D}_{V} W\right) f & =V^{\alpha}\left(\dot{\partial}_{\alpha} W^{\beta}\right)\left(\dot{\partial}_{\beta} f\right)+V^{\bar{\alpha}}\left(\dot{\partial}_{\bar{\alpha}} W^{\beta}\right)\left(\dot{\partial}_{\beta} f\right)+V^{\alpha}\left(\dot{\partial}_{\alpha} W^{\bar{\beta}}\right)\left(\dot{\partial}_{\bar{\beta}} f\right) \\
& +V^{\bar{\alpha}}\left(\dot{\partial}_{\bar{\alpha}} W^{\bar{\beta}}\right)\left(\dot{\partial}_{\bar{\beta}} f\right)+V^{\alpha} W^{\beta} C_{\beta \alpha}^{\gamma}\left(\dot{\partial}_{\gamma} f\right)+V^{\bar{\alpha}} W^{\bar{\beta}} C_{\bar{\alpha} \bar{\beta}}^{\bar{\gamma}}\left(\dot{\partial}_{\bar{\gamma}} f\right),
\end{aligned}
$$

hence the first identity is proved after a short computation. For the second one, (4) leads to $C_{\gamma \alpha}^{\varepsilon}=-h_{\gamma \bar{\sigma}}\left(\dot{\partial}_{\alpha} h^{\bar{\sigma} \varepsilon}\right)$, which we use to obtain

$$
\begin{aligned}
\mathcal{D}_{V}\left(\nabla^{v \bar{v}} f\right) & =\left[V^{\alpha} h^{\bar{\sigma} \gamma}\left(\dot{\partial}_{\alpha} \dot{\partial}_{\bar{\sigma}} f\right)+V^{\bar{\alpha}}\left(\dot{\partial}_{\bar{\alpha}} h^{\bar{\sigma} \gamma}\right)\left(\dot{\partial}_{\bar{\sigma}} f\right)+V^{\bar{\alpha}} h^{\bar{\sigma} \gamma}\left(\dot{\partial}_{\bar{\alpha}} \dot{\partial}_{\bar{\sigma}} f\right)\right] \mathcal{V}_{\gamma} \\
& +\left[V^{\alpha}\left(\dot{\partial}_{\alpha} h^{\bar{\sigma} \gamma}\right)\left(\dot{\partial}_{\gamma} f\right)+V^{\alpha} h^{\bar{\sigma} \gamma}\left(\dot{\partial}_{\alpha} \dot{\partial}_{\gamma} f\right)+V^{\bar{\alpha}} h^{\bar{\sigma} \gamma}\left(\dot{\partial}_{\bar{\alpha}} \dot{\partial}_{\gamma} f\right)\right] \mathcal{V}_{\bar{\sigma}}
\end{aligned}
$$

Therefore,

$$
\mathcal{G}\left(\mathcal{D}_{V}\left(\nabla^{v \bar{v}} f\right), W\right)=V W f-\left(\mathcal{D}_{V} W\right) f
$$

and the second identity is also proved.

\section{The warped product of algebroids}

We now consider two holomorphic Finsler algebroids, $\left(E_{1}, F_{1}\right)$ and $\left(E_{2}, F_{2}\right)$, where $E_{1}$ is a holomorphic vector bundle over the complex manifold $M_{1}$ and $E_{2}$ is a holomorphic vector bundle over the complex manifold $M_{2}$. We also consider their prolongations, $\mathcal{T} E_{1}$ and $\mathcal{T} E_{2}$, respectively, the Chern-Finsler connections, $\mathcal{D}^{1}$ and $\mathcal{D}^{2}$, and the bundle projections $\pi_{1}: E_{1} \rightarrow M_{1}$ and $\pi_{2}: E_{2} \rightarrow M_{2}$, respectively. Let $f: M_{1} \rightarrow \mathbb{R}_{+}$be a smooth function.

Inspired by [22], we use Aikou's definition [2] of a Finsler metric on a bundle to introduce the function $F: E_{1} \times E_{2} \rightarrow \mathbb{R}$ on the product bundle $E_{1} \times E_{2}$ by

$$
F\left(u_{1}, u_{2}\right)=F_{1}\left(u_{1}\right)+f^{2}\left(\pi_{1}\left(u_{1}\right)\right) F_{2}\left(u_{2}\right) .
$$

This is a Finsler metric function on the product bundle $E_{1} \times E_{2}$. Indeed, since $F_{1}$ and $F_{2}$ are smooth, it follows that $F$ is smooth on $\widetilde{E}_{1} \times \widetilde{E}_{2}$. As in the 
case of real Finsler manifolds [22], we restrict our study on $\widetilde{E}_{1} \times \widetilde{E}_{2}$, as $F$ is not necessarily smooth on $\left(u_{1}, 0\right)$ and $\left(0, u_{2}\right) \in E_{1} \times E_{2}$. The homogeneity property in the vector variables of $F$ is proved by the similar properties of $F_{1}$ and $F_{2}$, and the Hessian of $F$ with respect to the vector variables is:

$$
\left(\begin{array}{cc}
\dot{\partial}_{\alpha} \dot{\partial}_{\bar{\beta}} F_{1} & 0 \\
0 & f^{2} \dot{\partial}_{\alpha} \dot{\partial}_{\bar{\beta}} F_{2}
\end{array}\right)
$$

Since the Hessians of $F_{1}$ and $F_{2}$ are positive, it follows that the Hessian of $F$ is also positive.

We have thus defined the warped product of the algebroids $E_{1}$ and $E_{2}$ as the product bundle $E_{1} \times{ }_{f} E_{2}$, with the warping function $f$ and the Finsler metric $F=F\left(u_{1}, u_{2}\right)$. Therefore, $\left(E_{1} \times{ }_{f} E_{2}, F\right)$ is a complex Finsler bundle. In the following, we will make the construction on the prolongation for the warped product $E_{1} \times_{f} E_{2}$, namely its prolongation (as the product of the prolongations of the algebroids $E_{1}$ and $E_{2}$ ), adapted frames and the ChernFinsler connection.

Remark. In $[23,30]$ a doubly warped product is studied, with a more general definition,

$$
F\left(u_{1}, u_{2}\right)=g^{2}\left(\pi_{2}\left(u_{2}\right)\right) F_{1}\left(u_{1}\right)+f^{2}\left(\pi_{1}\left(u_{1}\right)\right) F_{2}\left(u_{2}\right) .
$$

The function $g$ is defined on $M_{2}$, while $f$ is defined on $M_{1}$. Still, the purpose of the present paper is to analyze the case of the warped product, therefore we leave the doubly warped product of algebroids to a later study.

\subsection{The prolongation bundle of the warped product bundle}

Consider two holomorphic Finsler algebroids, $\left(E_{1}, F_{1}\right)$ and $\left(E_{2}, F_{2}\right)$, and their warped product, $E_{1} \times_{f} E_{2}$, defined as above. Also, let $\mathcal{T} E_{1}$ and $\mathcal{T} E_{2}$ be the prolongations of $E_{1}$ and $E_{2}$, respectively, as defined in [17].

The vertical subbundles of the two prolongation bundles are defined using the projections $\tau_{i}: \mathcal{T} E_{i} \rightarrow E_{i}, \tau_{i}\left(e_{i}, v_{i}\right)=e_{i} \in E_{i}$, that is,

$$
V \mathcal{T} E_{i}=\operatorname{ker} \tau_{i}=\left\{\left(e_{i}, v_{i}\right) \in \mathcal{T} E_{i} \mid \tau_{i}\left(e_{i}, v_{i}\right)=0\right\},
$$

for $i=1,2$.

Since the prolongation bundle has similar properties to the tangent bundle of a manifold, as we have discussed in some previous papers $[16,17,18]$, we will further work in this setting instead of the tangent bundles of $E_{1}$ and $E_{2}$. We easily find that $\mathcal{T}\left(E_{1} \times E_{2}\right)=\mathcal{T} E_{1} \times \mathcal{T} E_{2}$. To be more precise, if we index by 1 and 2 the elements of the above construction of the prolongation of each 
of the algebroids $E_{1}$ and $E_{2}$, respectively, we can consider $\rho_{\mathcal{T}_{\times}}: \mathcal{T} E_{1} \times \mathcal{T} E_{2} \rightarrow$ $T E_{1} \times T E_{2}, \rho_{\mathcal{T}_{\times}}:=\rho_{\mathcal{T}_{1}} \times \rho_{\mathcal{T}_{2}}$, i.e.,

$$
\begin{aligned}
\rho_{\mathcal{T}_{\times}}\left(e_{1}, v_{1}, e_{2}, v_{2}\right) & =\left(\rho_{\mathcal{T}_{1}}\left(e_{1}, v_{1}\right), \rho_{\mathcal{T}_{2}}\left(e_{2}, v_{2}\right)\right) \\
& =\left(v_{1}, v_{2}\right) \in T\left(E_{1} \times E_{2}\right) \equiv T E_{1} \times T E_{2},
\end{aligned}
$$

where $\left(e_{1}, v_{1}, e_{2}, v_{2}\right) \in \mathcal{T} E_{1} \times \mathcal{T} E_{2}$. This is the anchor map of the prolongation algebroid $\mathcal{T}\left(E_{1} \times E_{2}\right) \equiv \mathcal{T} E_{1} \times \mathcal{T} E_{2}$ of the warped product $E_{1} \times_{f} E_{2}$.

If $p_{1}: \mathcal{T} E_{1} \times \mathcal{T} E_{2} \rightarrow \mathcal{T} E_{1}$ is the projection onto the first factor and $p_{2}:$ $\mathcal{T} E_{1} \times \mathcal{T} E_{2} \rightarrow \mathcal{T} E_{2}$ is the projection onto the second factor in the product of prolongations, then we can define the lifts of vector fields on $\mathcal{T} E_{1}$ or on $\mathcal{T} E_{2}$ to $\mathcal{T} E_{1} \times \mathcal{T} E_{2}$ as follows. The lift of $U_{1} \in \Gamma\left(\mathcal{T} E_{1}\right)$ on $\mathcal{T} E_{1} \times \mathcal{T} E_{2}$ is $\widehat{U}_{1} \in \Gamma\left(\mathcal{T} E_{1} \times \mathcal{T} E_{2}\right)$ which satisfies the conditions $p_{1}\left(\widehat{U}_{1}\right)=U_{1}, p_{2}\left(\widehat{U}_{1}\right)=0$. Similarly, the lift of $U_{2} \in \Gamma\left(\mathcal{T} E_{2}\right)$ on $\mathcal{T} E_{1} \times \mathcal{T} E_{2}$ is $\widehat{U}_{2} \in \Gamma\left(\mathcal{T} E_{1} \times \mathcal{T} E_{2}\right)$ which satisfies the conditions $p_{1}\left(\widehat{U}_{2}\right)=0, p_{2}\left(\widehat{U}_{2}\right)=U_{2}$.

Furthermore, $\operatorname{ker}\left(\tau_{1} \times \tau_{2}\right)=\operatorname{ker} \tau_{1} \oplus \operatorname{ker} \tau_{2}$, hence $V \mathcal{T} E_{1} \times V \mathcal{T} E_{2}$ is the vertical bundle of $\mathcal{T} E_{1} \times \mathcal{T} E_{2}$. With respect to the Chern-Finsler nonlinear connections of $\mathcal{T} E_{1}$ and $\mathcal{T} E_{2}$, we obtain the following decomposition:

$$
\mathcal{T}\left(E_{1} \times E_{2}\right)=\mathcal{T} E_{1} \oplus \mathcal{T} E_{2}=H \mathcal{T} E_{1} \oplus V \mathcal{T} E_{1} \oplus H \mathcal{T} E_{2} \oplus V \mathcal{T} E_{2} .
$$

Let us denote by $N_{\alpha}^{\beta}=\stackrel{1}{N_{\alpha}^{\beta}}\left(z_{1}, u_{1}\right)$ and $\stackrel{2}{N_{\alpha}^{\beta}}=\stackrel{2}{N_{\alpha}^{\beta}}\left(z_{2}, u_{2}\right)$, the coefficients of the Chern-Finsler nonlinear connections on $\mathcal{T} E_{1}$ and $\mathcal{T} E_{2}$, respectively. Also,

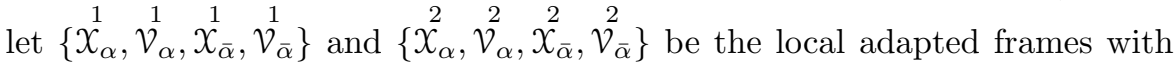
respect to the above connections on $\mathcal{T} E_{1}$ and $\mathcal{T} E_{2}$, respectively. It is obvious that the Lie brackets $[\cdot, \cdot]_{\mathcal{T}_{\times}}$on $\mathcal{T} E_{1} \times \mathcal{T} E_{2}$ satisfy the identities:

$$
\begin{aligned}
& {\left[U_{1}, V_{2}\right]_{\mathcal{T}_{\times}}=0,} \\
& {\left[U_{1}, V_{1}\right]_{\mathcal{T}_{\times}}=\left[U_{1}, V_{1}\right]_{\mathcal{T} E_{1}},} \\
& {\left[U_{2}, V_{2}\right]_{\mathcal{T}_{\times}}=\left[U_{2}, V_{2}\right]_{\mathcal{T} E_{2}},}
\end{aligned}
$$

for all $U_{1}, V_{1} \in \Gamma\left(\mathcal{T} E_{1}\right)$ and $U_{2}, V_{2} \in \Gamma\left(\mathcal{T} E_{2}\right)$.

\subsection{The Chern-Finsler connection of the warped product}

As in the case of Finsler manifolds [22], the Koszul formula plays an important role in the study of the relation between the connections on each prolongation bundle and the connection on their product. Since, in our case, the formula (6) holds only for vertical fields on a prolongation bundle, we will further work only on the vertical part of the above decomposition, which we denote by $V \mathcal{T}=V \mathcal{T} E_{1} \oplus V \mathcal{T} E_{2}$. If $\mathcal{G}_{1}$ and $\mathcal{G}_{2}$ are two Hermitian metrics given as in 
(5) on $\mathcal{T} E_{1}$ and $\mathcal{T} E_{2}$, respectively, then we use their restrictions $\mathcal{G}_{1}^{v}$ and $\mathcal{G}_{2}^{v}$ on the vertical bundles to define a Hermitian metric $\mathcal{G}$ on $V \mathcal{T}$ by

$$
\mathcal{G}^{v}(\cdot, \cdot)=\mathcal{G}_{1}^{v}(\cdot, \cdot)+f^{2}\left(\pi_{1}\left(v_{1}\right)\right) \mathcal{G}_{2}^{v}(\cdot, \cdot) .
$$

Similar reasons as in the case of real warped Finsler manifolds [22] lead to the following result.

Theorem 1. Let $U_{1}, V_{1} \in V \mathcal{T} E_{1}$ and $U_{2}, V_{2} \in V \mathcal{T} E_{2}$. Then:

1. $\mathcal{D}_{U_{1}} V_{1}$ on $V \mathcal{T} E_{1} \oplus V \mathcal{T} E_{2}$ is the lift of $\mathcal{D}_{U_{1}} V_{1}$ on $V \mathcal{T} E_{1}$;

2. $\mathcal{D}_{U_{1}} U_{2}=\mathcal{D}_{U_{2}} U_{1}=\left(U_{2} f / f\right) U_{1}$;

3. $\mathcal{D}_{U_{2}} V_{2}=\mathcal{D}_{U_{2}}^{2} V_{2}-\frac{\mathcal{G}\left(U_{2}, V_{2}\right)}{f} \nabla^{v \bar{v}} f$;

4. $\mathcal{T}\left(U_{1}, U_{2}\right)=\mathfrak{T}\left(U_{2}, U_{1}\right)=0$;

Proof. The line of the proof from [22] can be easily followed here for vector fields on the vertical subbundles of the two prolongation algebroids, $\mathcal{T} E_{1}$ and $\mathcal{T} E_{2}$, respectively.

We are now interested in the local description of the previous result. First, we have

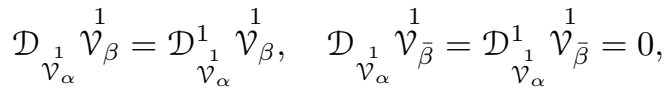

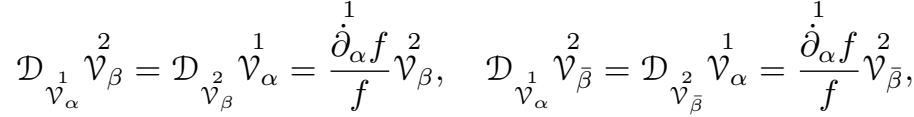

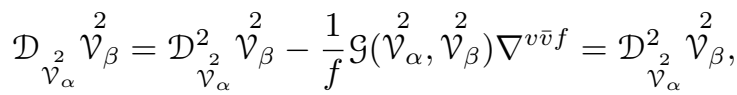

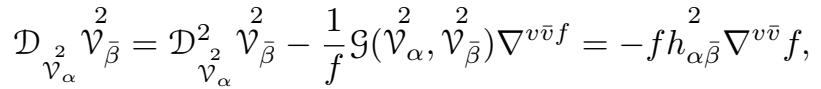

together with their conjugates. For the last two identities, we used $\mathcal{G}\left(\mathcal{V}_{\alpha}^{2}, \mathcal{V}_{\beta}^{2}\right)=$ 0 , since $\mathcal{G}$ is Hermitian, and $\mathcal{G}\left(\mathcal{V}_{\alpha}^{2}, \mathcal{V}_{\bar{\beta}}^{2}\right)=f^{2} h_{\alpha \bar{\beta}}$. 
Therefore, if we denote

$$
\begin{aligned}
& \mathcal{D}_{\mathcal{V}_{\alpha}^{1}}{\stackrel{1}{\mathcal{V}^{\prime}}}_{\beta}=\stackrel{11,1}{C}_{\beta \alpha}^{\gamma} \mathcal{V}_{\gamma}^{1}+\stackrel{11, \overline{1}}{C}_{\beta \alpha}^{\bar{\sigma}} \mathcal{V}_{\bar{\sigma}}^{1}+\stackrel{11,2}{C}_{\beta \alpha}^{\gamma} \mathcal{V}_{\gamma}^{2}+\stackrel{11, \overline{2}}{C}_{\beta \alpha}^{\bar{\sigma}} \mathcal{V}_{\bar{\sigma}}^{2}, \\
& \mathcal{D}_{\mathcal{V}_{\alpha}^{1}} \mathcal{V}_{\bar{\beta}}^{1}=\stackrel{\overline{1} 1,1}{C} \frac{\gamma}{\bar{\beta} \alpha} \mathcal{V}_{\gamma}+\stackrel{\overline{1} 1, \overline{1}}{C} \frac{\bar{\sigma}}{\bar{\beta} \alpha} \mathcal{V}_{\bar{\sigma}}+\stackrel{\overline{1} 1,2}{C} \frac{\gamma}{\bar{\beta} \alpha} \mathcal{V}_{\gamma}^{2}+\stackrel{\overline{1} 11, \overline{2}}{C} \frac{\bar{\sigma}}{\bar{\beta} \alpha} \mathcal{V}_{\bar{\sigma}}^{2}, \\
& \mathcal{D}_{\mathcal{V}_{\alpha}^{1}} \mathcal{V}_{\beta}^{2}=\stackrel{21,1}{C}_{\beta \alpha}^{\gamma} \mathcal{V}_{\gamma}^{1}+\stackrel{21, \overline{1}}{C}_{\beta \alpha}^{\bar{\sigma}} \mathcal{V}_{\bar{\sigma}}^{1}+\stackrel{21,2}{C}_{\beta \alpha}^{\gamma} \mathcal{V}_{\gamma}^{2}+\stackrel{21,}{C}_{\beta \alpha}^{\bar{\sigma}} \mathcal{V}_{\bar{\sigma}}^{2}, \\
& \mathcal{D}_{\mathcal{V}_{\alpha}^{1}} \mathcal{V}_{\bar{\beta}}^{2}=\stackrel{\overline{2} 1,1}{C}{ }_{\bar{\beta} \alpha}^{\gamma} \mathcal{V}_{\gamma}+\stackrel{\overline{2} 1, \overline{1}}{C} \frac{\bar{\sigma}}{\bar{\beta} \alpha} \mathcal{V}_{\bar{\sigma}}+\stackrel{\overline{2} 1,2}{C}{ }_{\bar{\beta} \alpha}^{\gamma} \mathcal{V}_{\gamma}^{2}+\stackrel{\overline{2} 1, \overline{2}}{C} \frac{\bar{\sigma}}{\bar{\beta} \alpha} \mathcal{V}_{\bar{\sigma}}^{2}, \\
& \mathcal{D}_{\mathcal{V}_{\alpha}^{2}} \mathcal{V}_{\beta}^{2}=\stackrel{22}{C}_{\beta \alpha}^{\gamma} \mathcal{V}_{\gamma}+\stackrel{22, \overline{1}}{C}_{\beta \alpha}^{\bar{\sigma}} \mathcal{V}_{\bar{\sigma}}^{1}+\stackrel{22,2}{C}_{\beta \alpha}^{\gamma} \mathcal{V}_{\gamma}^{2}+\stackrel{22, \overline{2}}{C}_{\beta \alpha}^{\bar{\sigma}} \mathcal{V}_{\bar{\sigma}}^{2} \text {, } \\
& \mathcal{D}_{\mathcal{V}_{\alpha}^{2}} \mathcal{V}_{\bar{\beta}}^{2}=\stackrel{\overline{2} 2,1}{C}{ }_{\bar{\beta} \alpha}^{\gamma} \mathcal{V}_{\gamma}+\stackrel{\overline{2} 2, \overline{1}}{C} \frac{\bar{\sigma}}{\bar{\beta} \alpha} \mathcal{V}_{\bar{\sigma}}+\stackrel{\overline{2} 2,2}{C} \frac{\gamma}{\bar{\beta} \alpha} \mathcal{V}_{\gamma}^{2}+\stackrel{\overline{2} 2, \overline{2}}{C} \frac{\bar{\sigma}}{\bar{\beta} \alpha} \mathcal{V}_{\bar{\sigma}}^{2},
\end{aligned}
$$

we obtain

$$
\begin{aligned}
& \stackrel{11,1}{C}{ }_{\beta_{1} \alpha_{1}}^{\gamma_{1}}=\stackrel{1}{C}_{\beta_{1} \alpha_{1}}^{\gamma_{1}}=\stackrel{1}{h}^{\bar{\sigma}_{1} \gamma_{1}}\left(\dot{\leftrightarrow}_{\alpha_{1}} \stackrel{1}{h}_{\beta_{1} \bar{\sigma}_{1}}\right) \\
& \stackrel{21,2}{C} \underset{\beta_{2} \alpha_{1}}{\gamma_{2}}=\delta_{\beta_{2}}^{\gamma_{2}} \frac{1}{\dot{\partial}_{\alpha_{1}} f}, \quad \stackrel{\overline{2} 1, \overline{2}}{C}{\overline{\bar{\sigma}_{2} \alpha_{1}}}_{\bar{\beta}_{2}}=\delta_{\bar{\beta}_{1}}^{\bar{\gamma}_{1}} \frac{\dot{\partial}_{\bar{\alpha}_{1}} f}{f}, \\
& \left.\stackrel{22,2}{C} \underset{\beta_{2} \alpha_{2}}{\gamma_{2}}=\stackrel{2}{C}_{\beta_{2} \sigma_{2}}^{\gamma_{2}}=\stackrel{2}{\bar{h}^{\bar{\sigma}_{2} \gamma_{2}}} \stackrel{2}{\dot{\partial}_{\alpha_{2}}} \stackrel{2}{h}_{\beta_{2} \bar{\sigma}_{2}}\right), \\
& \stackrel{\overline{2} 2,1}{C}{ }_{\bar{\beta}_{2} \alpha_{2}}^{\gamma_{1}}=-f\left(\dot{\partial}_{\bar{\sigma}} f\right) h^{\bar{\sigma} \gamma} \stackrel{2}{h}_{\alpha \bar{\beta}}, \\
& \stackrel{\overline{2} 2, \overline{1}}{C}{ }_{\bar{\beta}_{2} \alpha_{2}}=-f\left(\dot{\partial}_{\gamma} f\right) h^{\bar{\sigma} \gamma} \stackrel{2}{h}_{\alpha \bar{\beta}} .
\end{aligned}
$$

All the other coefficients are zero.

The curvature of the product $\mathcal{T} E_{1} \times \mathcal{T} E_{2}$ is defined as usual by $\mathcal{R}(U, V) W=$ $\mathcal{D}_{U} \mathcal{D}_{V} W-\mathcal{D}_{V} \mathcal{D}_{U} W-\mathcal{D}_{[U, V]} W$.

The connection between the curvature of the warped product and the curvatures of each prolongation bundle in the product is given in the following

Theorem 2. If $\mathcal{T} E_{1} \times \mathcal{T} E_{2}$ is the product of the prolongations of two holomorphic vector bundles, with the curvatures $\mathcal{R}$ on the product bundle and $\mathcal{R}^{1}$ and $\mathcal{R}^{2}$ on $\mathcal{T} E_{1}$ and $\mathcal{T} E_{2}$, respectively, then for $U_{1}, V_{1}, W_{1} \in V \mathcal{T} E_{1}$ and $U_{2}, V_{2}, W_{2} \in V \mathcal{T} E_{2}$, the following identities hold:

1. $\mathcal{R}\left(U_{1}, V_{1}\right) W_{1}$ is the lift of $\mathcal{R}^{1}\left(U_{1}, V_{1}\right) W_{1}$; 


$$
\begin{aligned}
& \text { 2. } \mathcal{R}\left(V_{2}, U_{1}\right) V_{1}=-\frac{1}{f}\left[\mathcal{H} f\left(U_{1}, V_{1}\right)-\left(\mathcal{D}_{U_{1}^{v}} V_{1}^{v}-\mathcal{D}_{U_{1}^{\bar{v}}} V_{1}^{\bar{v}}\right) f\right] V_{2} ; \\
& \text { 3. } \mathcal{R}\left(V_{2}, W_{2}\right) U_{1}=0 ; \\
& \text { 4. } \mathcal{R}\left(V_{2}, W_{2}\right) U_{2}=\mathcal{R}^{2}\left(V_{2}, W_{2}\right) U_{2}+\frac{\mathcal{G}\left(\nabla^{v \bar{v}} f, \nabla^{v \bar{v}} f\right)}{f^{2}}\left(\mathcal{G}\left(V_{2}, U_{2}\right) W_{2}-\mathcal{G}\left(W_{2}, U_{2}\right) V_{2}\right)
\end{aligned}
$$

Proof. The first statement is obvious.

For the second identity, we compute $\mathcal{R}\left(V_{2}, U_{1}\right) V_{1}=\mathcal{D}_{V_{2}} \mathcal{D}_{U_{1}} V_{1}-\mathcal{D}_{U_{1}} \mathcal{D}_{V_{2}} V_{1}-$ $\mathcal{D}_{\left[V_{2}, U_{1}\right]} V_{1}$. First, $\left[V_{2}, U_{1}\right]=0$. Then, since $\mathcal{D}_{U_{1}} V_{1} \in V \mathcal{T} E_{1}$, from Theorem 1 we obtain $\mathcal{D}_{V_{2}} \mathcal{D}_{U_{1}} V_{1}=\frac{1}{f}\left(\mathcal{D}_{U_{1}} V_{1}\right) f V_{2}$. Further, we have:

$$
\begin{aligned}
\mathcal{D}_{U_{1}} \mathcal{D}_{V_{2}} V_{1} & =\mathcal{D}_{U_{1}}\left[\left(\frac{V_{1} f}{f}\right) V_{2}\right]=U_{1}\left(\frac{V_{1} f}{f}\right) V_{2}+\frac{V_{1} f}{f} \mathcal{D}_{U_{1}} V_{2} \\
& =\frac{1}{f}\left(U_{1} V_{1} f\right) V_{2}+\left(V_{1} f\right) U_{1}\left(\frac{1}{f}\right) V_{2}+\frac{1}{f^{2}}\left(V_{1} f\right)\left(U_{1} f\right) V_{2} \\
& =\frac{1}{f}\left(U_{1} V_{1} f\right) V_{2}-\frac{1}{f^{2}}\left(V_{1} f\right)\left(U_{1} f\right) V_{2}+\frac{1}{f^{2}}\left(V_{1} f\right)\left(U_{1} f\right) V_{2} \\
& =\frac{1}{f}\left(U_{1} V_{1} f\right) V_{2},
\end{aligned}
$$

where we have used again Theorem 1. Hence, due to (9),

$$
\begin{aligned}
\mathcal{R}\left(V_{2}, U_{1}\right) V_{1} & =\frac{1}{f}\left[\left(\mathcal{D}_{U_{1}} V_{1}\right) f-U_{1} V_{1} f\right] V_{2} \\
& =\left[\mathcal{H}^{f}\left(U_{1}, V_{1}\right)-\left(\mathcal{D}_{U_{1}^{v}} V_{1}^{v}-\mathcal{D}_{U_{1}^{\bar{v}}} V_{1}^{\bar{v}}\right) f\right] V_{2} .
\end{aligned}
$$

For the third identity, we have $\mathcal{R}\left(V_{2}, W_{2}\right) U_{1}=\mathcal{D}_{V_{2}} \mathcal{D}_{W_{2}} U_{1}-\mathcal{D}_{W_{2}} \mathcal{D}_{V_{2}} U_{1}$, since $\left[V_{2}, W_{2}\right]=0$. From

$$
\mathcal{D}_{V_{2}} \mathcal{D}_{W_{2}} U_{1}=\mathcal{D}_{V_{2}}\left[\left(\frac{U_{1} f}{f}\right) W_{2}\right]=V_{2}\left(\frac{U_{1} f}{f}\right) W_{2}+\frac{U_{1} f}{f} \mathcal{D}_{V_{2}} W_{2}
$$

and

$$
\mathcal{D}_{W_{2}} \mathcal{D}_{V_{2}} U_{1}=\mathcal{D}_{W_{2}}\left[\left(\frac{U_{1} f}{f}\right) V_{2}\right]=W_{2}\left(\frac{U_{1} f}{f}\right) V_{2}+\frac{U_{1} f}{f} \mathcal{D}_{W_{2}} V_{2},
$$

since $V_{2}\left(\frac{U_{1} f}{f}\right)=W_{2}\left(\frac{U_{1} f}{f}\right)=0$, we get

$$
\mathcal{R}\left(V_{2}, W_{2}\right) U_{1}=\frac{1}{f}\left(U_{1} f\right) \mathcal{T}\left(V_{2}, W_{2}\right)=0
$$

as the vertical torsion vanishes.

The last identity is obtained after a straightforward computation using the properties from Theorem 1, following similar ideas from [22]. 


\section{A warped Finsler model for gravitation and electro- magnetism}

We give a basic example of warped product with applications in theoretical physics. As known from unification theories of gravity and electromagnetism, we need higher dimensional spaces (of dimension 5 in Kaluza-Klein theories or 8 in Yang-Mills theories, etc.) with adequate metric tensors. For such theories, product spaces are the most useful geometrical settings.

Let $M$ be the complexified space-time considered in [29], $\operatorname{dim}_{\mathbb{C}} M=2$, and take $\left(z^{1}, z^{2}\right)$ complex coordinates on $M$. We consider $E_{1}=T^{\prime} M$, the holomorphic tangent bundle, which has a 4-dimensional complex manifold structure, with $\left(z^{1}, z^{2}, \eta^{1}, \eta^{2}\right)$ complex coordinates on $T^{\prime} M$. The bundle $T^{\prime} M$ has a natural structure of holomorphic Lie algebroid, with the anchor map $\rho_{1}$ the identity. A complex Finsler function $F_{1}: T^{\prime} M \rightarrow \mathbb{R}_{+}$is the weakly gravitational metric considered in [29]:

$F_{1}:=\left(1+\frac{2 \Phi}{c^{2}}\right)\left|\eta^{1}\right|^{2}-i\left(1-\frac{2 \Phi}{c^{2}}\right) \eta^{1} \bar{\eta}^{2}+i\left(1-\frac{2 \Phi}{c^{2}}\right) \eta^{2} \bar{\eta}^{1}-\left(1-\frac{2 \Phi}{c^{2}}\right)\left|\eta^{2}\right|^{2}$

where $\Phi$ is a smooth 0 -homogeneous function in $\eta$ on $T^{\prime} M$, with the physical meaning of a complex gravitational potential, satisfying $\Phi>\frac{c^{2}}{2}, c \in \mathbb{R}^{*}$.

The metric tensor is

$$
\stackrel{1}{h}_{j \bar{k}}(z, \eta)=\left(\begin{array}{cc}
1+\frac{2 \Phi}{c^{2}} & -i\left(1-\frac{2 \Phi}{c^{2}}\right) \\
i\left(1-\frac{2 \Phi}{c^{2}}\right) & -\left(1-\frac{2 \Phi}{c^{2}}\right)
\end{array}\right), \quad j, k=1,2 \quad \text { and } \quad i:=\sqrt{-1},
$$

while its inverse,

$$
\stackrel{1}{h}^{k} j(z, \eta)=\left(\begin{array}{cc}
\frac{1}{2} & -\frac{i}{2} \\
\frac{i}{2} & -\frac{1+\frac{2 \Phi}{c^{2}}}{2\left(1-\frac{2 \Phi}{c^{2}}\right)}
\end{array}\right), \quad j, k=1,2 .
$$

As stated in [29], this metric is Finsler under the assumptions:

i) $\Phi>\frac{c^{2}}{2}$, i.e. $\operatorname{det}\left(\stackrel{1}{h}_{i \bar{j}}\right)>0$;

ii) $\Phi$ is 0-homogeneous with respect to $\eta$;

iii) $i \Phi_{\cdot 2}=\Phi_{.1}$, where $\Phi_{\cdot h}=\frac{\partial \Phi}{\partial \eta^{h}}, h=1,2$.

The components of the Chern-Finsler nonlinear connection are

$$
\stackrel{1}{N}_{k}^{1}=0, \quad \stackrel{1}{N}_{k}^{2}=\frac{-2 i}{c^{2}\left(1-\frac{2 \Phi}{c^{2}}\right)}\left(\eta^{1}-i \eta^{2}\right) \Phi_{k} .
$$


For the second holomorphic Lie algebroid, we take again the complexified space-time $M$, and on $E_{2}=T^{\prime} M$ we can take another Finsler metric, proposed in [32]:

$$
F_{2}:=F_{0}^{2}+\sigma(z)|\beta|^{2}
$$

where:

i) $F_{0}$ is a given Finsler metric (for instance the weakly gravitational metric $\left.F_{1}\right)$ on $M$;

ii) $\sigma: M \rightarrow \mathbb{R}$ is a function which satisfies $\sigma(z) \geq-\frac{F_{0}^{2}}{|\beta|^{2}}(z, \eta)$ for all $(z, \eta) \in$ $T^{\prime} M$

iii) $\beta=B_{k}(z) \eta^{k}$ is the Beil holomorphic form; usually, $B_{k}(z)$ are the components of an electromagnetic field.

The metric tensor is given in this case by

$$
\stackrel{2}{h}_{i \bar{j}}=g_{i \bar{j}}+\sigma(z) B_{i}(z) B_{\bar{j}}(z),
$$

where $g_{i \bar{j}}$ is the metric of $\left(M, F_{0}\right)$ and $B_{\bar{j}}=\overline{B_{j}}$. The inverse of $(15)$ is

$$
\stackrel{2}{h}^{j i}=g^{\bar{j} i}-\frac{\sigma}{1+\sigma \mathbb{B}^{2}},
$$

where $\mathbb{B}=g_{i \bar{j}} B^{i} B^{\bar{j}}$. The coefficients of the Chern-Finsler nonlinear connection are $\stackrel{2}{k}_{k}^{j}=N_{k}^{j}+A_{k}^{j}$, where $A_{k}^{j}=\stackrel{2}{h^{\bar{m} j}} \partial_{k}\left(\sigma B_{l} B_{\bar{m}}\right) \eta^{l}$.

The prolongation of a Lie algebroid is a pull-back bundle of $T^{\prime} E$ on $E$ by the ancor map, that is, $\rho_{*}^{-1} T^{\prime} E$. It follows that, if $E$ is in particular $T^{\prime} M$ and $\rho=i d$, then the holomorphic prolongation $\mathcal{T}^{\prime} T^{\prime} M$ of the holomorphic algebroid $T^{\prime} M$ is canonically isomorphic to $T^{\prime} T^{\prime} M$, with complex coordinates $\left(z^{k}, \eta^{k}, x^{k}, \theta^{k}\right), k=1,2$, where $\eta^{k}, x^{k}$ both change by the matrix $\frac{\partial z^{\prime k}}{\partial z^{j}}$. This remark can be found in [24] for the real case. More precisely, since $\rho=\mathrm{id}$, the definition of the holomorphic prolongation bundle is in this case

$$
\mathcal{T}^{\prime}\left(T^{\prime} M\right)=\left\{(x, v) \in T^{\prime} M \times T^{\prime}\left(T^{\prime} M\right) \mid \operatorname{id}(x)=\pi_{*}^{\prime}(v)\right\} .
$$

Hence, for the complex coordinates $\left(z^{k}, \eta^{k}\right)$ on $T^{\prime} M$, we take $x=x^{k} \frac{\partial}{\partial z^{k}} \in$ $T^{\prime} M$ and $v=v^{k} \frac{\partial}{\partial z^{k}}+\theta^{k} \frac{\partial}{\partial \eta^{k}} \in T_{u}^{\prime}\left(T^{\prime} M\right)$ a vector tangent to $T^{\prime} M$ at $u=$ $\eta^{k} \frac{\partial}{\partial z^{k}} \in T^{\prime} M$. We have $\pi_{*}^{\prime}(v)=v^{k} \frac{\partial}{\partial z^{k}}$, thus $v^{k}=x^{k}$ and the vector $v \in$ $\mathcal{T}^{\prime}\left(T^{\prime} M\right)$ is of the form $v=x^{k} \frac{\partial}{\partial z^{k}}+\theta^{k} \frac{\partial}{\partial \eta^{k}}$. 
The local basis of holomorphic sections in $\Gamma\left(\mathcal{T}_{u}^{\prime} T^{\prime} M\right)$ is $\left\{z_{k}(u)=\left(\left.\frac{\partial}{\partial z^{k}}\right|_{z}\right.\right.$, $\left.\left.\left.\frac{\partial}{\partial z^{k}}\right|_{u}\right), v_{k}(u)=\left(0,\left.\frac{\partial}{\partial \eta^{k}}\right|_{u}\right)\right\}$. We take $x_{k}=z_{k}-N_{k}^{j} \mathcal{v}_{j}$, therefore, the adapted frame on $\mathcal{T}^{\prime} T^{\prime} M$ is $\left\{X_{k}, \mathcal{V}_{k}\right\}$, where $\left\{X_{k}\right\}$ is a basis of the horizontal bundle $H \mathcal{T}^{\prime} T^{\prime} M$ and $\left\{\mathcal{V}_{k}\right\}$ is a basis of the vertical bundle $V \mathcal{T}^{\prime} T^{\prime} M$. We have the isomorphic identifications $z_{k} \equiv \frac{\partial}{\partial z_{k}}, \nu_{k} \equiv \frac{\partial}{\partial \eta_{k}}$ and $X_{k} \equiv \frac{\delta}{\delta z_{k}}=$ $\frac{\partial}{\partial z^{k}}-N_{k}^{j}(z, \eta) \frac{\partial}{\partial \eta^{j}}$ and by complexification we obtain that $\mathcal{T} T^{\prime} M \equiv T_{\mathbb{C}}\left(T^{\prime} M\right)$. We can apply these considerations in our example for $E_{1}=E_{2}=T^{\prime} M$.

Further, we consider a holomorphic function $f: M \rightarrow \mathbb{R}$ and on the warped product $\mathcal{T} T^{\prime} M \times_{f} \mathcal{T} T^{\prime} M \equiv T_{\mathbb{C}}\left(T^{\prime} M\right) \times_{f} T_{\mathbb{C}}\left(T^{\prime} M\right)$ we define the Finsler metric

$$
F(z, \eta)=F_{1}(z, \eta)+f^{2}(z) F_{2}(z, \eta),
$$

which offers a possible model for unification theories of gravitation and electromagnetism, depending on two functions on $M, f$ and $\sigma$. It might be of interest for a future study the case when $F_{0}=F_{1}$ and, moreover, $f(z)=\sigma(z)$.

Let us also note that other interesting applications might be found in the setting $E_{1}=T^{\prime} M$ endowed with a Lagrangian and $E_{2}=T^{\prime *} M$ endowed with a Hamiltonian [29].

\section{Acknowledgements.}

The authors are indebted to the anonymous referee for the suggestions and comments that helped them improve this article.

\section{References}

[1] L. Abrunheiro, L. Colombo, Lagrangian Lie subalgebroids generating dynamics for second-order mechanical systems on Lie algebroids, Mediterr. J. Math. 15, No. 2, Paper No. 57, 19 p. (2018).

[2] T. Aikou, Finsler geometry on complex vector bundles, MSRI Publications 50, 83-105 (2004).

[3] S.B. Alexander, R.L. Bishop, Warped products of Hadamard spaces, Manuscr. Math. 96, No. 4, 487-505 (1998).

[4] G.S. Asanov, Finslerian metric functions over the product $\mathbb{R} \times M$ and their potential applications, Rep. Math. Phys. 41, No. 1, 117-132 (1998).

[5] A. Bejancu, On the theory of Finsler submanifolds, Meetings of Minds, ed. P.L. Antonelli, Kluwer Acad. Press, 111-129 (1999). 
[6] B.-Y. Chen, Geometry of warped product CR-submanifolds in Kähler manifolds, Monatsh. Math. 133, 177-195 (2001).

[7] C.-H. Chen, Warped products of metric spaces of curvature bounded from above, Trans. Am. Math. Soc. 351, No. 12, 4727-4740 (1999).

[8] S. S. Chern, Z. Shen, Riemann-Finsler Geometry, Singapore: World Scientific (2005)

[9] L. Colombo, Second-order constrained variational problems on Lie algebroids: Applications to optimal control, J. Geom. Mech. 9, No. 1, 1-45 (2017).

[10] K. Grabowska, J. Grabowski, Tulczyjew triples: from statics to field theory, J. Geom. Mech. 5, No. 4, 445-472 (2013).

[11] K. Grabowska, J. Grabowski, P. Urbanski, Geometrical mechanics on algebroids, Int. J. of Geom. Methods in Mod. Phys. 3, 559-575 (2006).

[12] P. Higgins, K. C. H. Mackenzie, Algebraic constructions in the cathegory of Lie algebroids, J. Algebra 129, No. 1, 194-230 (1990).

[13] S.-T. Hong, Warped product and FRW Cosmology, J. of the Korean Phys. Soc., Vol. 45, 95-98 (2004).

[14] S.-T. Hong, Warped products and black holes, Nuovo Cimento Soc. Ital. Fis. B 120, 1227-1232 (2005).

[15] C. Ida, P. Popescu, On Almost Complex Lie Algebroids, Mediterr. J. Math. 13, No. 2, 803-824 (2016).

[16] A. Ionescu, On holomorphic Lie algebroids, Bull. Transilv. Univ. Braşov, Ser. III, Math. Inform. Phys. 9(58), No. 1, 53-66 (2016).

[17] A. Ionescu, Finsler structures on holomorphic Lie algebroids, Novi Sad J. Math., Vol. 47, No. 2, 117-132 (2017).

[18] A. Ionescu, Laplace operators on holomorphic Lie algebroids, An. Şt. Univ. Ovidius, Constanţa, Vol. 26(1), 141-158 (2018).

[19] A. Ionescu, G. Munteanu, Connections on the total space of a holomorphic Lie algebroid, Mediterr. J. Math. 14, No. 4, Paper No. 163, 23 p. (2017).

[20] M. Jozwikowski, Prolongations vs. Tulczyjew triples in Geometric Mechanics, arXiv:1712.09858 [math-ph]. 
[21] J. Klein, Espaces variationelles et mécaniques, Ann. Inst. Fourier 12 (1962), 1-124.

[22] L. Kozma, I. R. Peter, C. Varga, Warped product of Finsler manifolds, Ann. Univ. Sci. Budap., Rolando Eötvös, Sect. Math. 44, 157-170 (2001).

[23] L. Kozma, I. R. Peter, C. Varga, Doubly warped product of Finsler manifolds, Miron, Radu (ed.), Lagrange and Hamilton geometries and their applications, Bucharest: Fair Partners Publishers (ISBN 973-8470-293/pbk). Handbooks. Treatises. Monographs 49, 145-152 (2004).

[24] M. de León, J. C. Marrero, E. Martinez, Lagrangian submanifolds and dynamics on Lie algebroids, J. Phys. A, Math. Gen. 38, No. 24, R241R308 (2005).

[25] E. Martinez, Geometric formulation of mechanics on Lie algebroids, Publ. R. Soc. Mat. Esp. 2, 209-222 (1999).

[26] E. Martinez, Lagrangian mechanics on Lie algebroids, Acta Appl. Math. 67 , No. 3, 295-320 (2001).

[27] E. Martinez, Lie algebroids in classical mechanics and optimal control, SIGMA, Symmetry Integrability Geom. Methods Appl. 3, Paper 050, 17 p. (2007).

[28] G. Munteanu, Complex spaces in Finsler, Lagrange and Hamilton geometries, Kluwer Acad. Publishers, Dordrecht, 2004.

[29] G. Munteanu, N. Aldea, A Complex Finsler Approach of Gravity, Int. J. of Geom. Methods in Mod. Phys. 9, No. 7, Paper No. 1250058, 16 p. (2012).

[30] E. Peyghan, A. Tayebi, On doubly warped product Finsler manifolds, Nonlinear. Anal., Real World Appl. 13, No. 4, 1703-1720 (2012).

[31] E. Peyghan, A. Tayebi, L. Nourmohammadi, On Twisted Products Finsler Manifolds, ISRN Geom. 2013, Article ID 732432, 12 p. (2013).

[32] A. Szász, Beil metrics in complex Finsler geometry, Balkan J. Geom. Appl. 20, No. 2, 72-83 (2015).

[33] M. Ulanovskii, Lorentzian warped product and singularity, Gen. Relativ. Gravitation 31, No. 12, 1813-1820 (1999).

[34] A. Weinstein, Lagrangian mechanics and grupoids, Fields Inst. Comm. 7, 206-231 (1996). 
[35] Z. Wu, C. Zhong, Some results on product complex Finsler manifolds, Acta Math. Sci., Ser. B, Engl. Ed. 31, No. 4, 1541-1552 (2011).

Alexandru IONESCU,

Department of Mathematics and Informatics,

Transilvania University of Braşov,

Bdul Iuliu Maniu 50, 500091 Braşov, Romania.

Email: alexandru-codrin.ionescu@unitbv.ro

Gheorghe MUNTEANU,

Department of Mathematics and Informatics,

Transilvania University of Braşov,

Bdul Iuliu Maniu 50, 500091 Braşov, Romania.

Email: gh.munteanu@unitbv.ro 